\title{
Fulfilling the Potential of Point-of-Care Ultrasound in Hospital Medicine
}

\author{
Molly F Wyman, MD', Matthew D Yocum, MD, Daniel J Schnobrich, MD*
}

Divisions of General Internal Medicine and Hospital Pediatrics, University of Minnesota, Minneapolis, Minnesota.

red he enthusiasm surrounding point-of-care ultrasound (POCUS) is clear and well founded. POCUS is a powerful tool that produces valuable diagnostic information for common and important clinical problems faced by hospitalists, such as pneumonia, soft-tissue infections, ${ }^{1}$ and myriad other applications. It can inform the evaluation and management of complex clinical problems such as dyspnea. ${ }^{2}$ Beyond its diagnostic potential, POCUS is well known to improve common procedures performed by adult and pediatric hospitalists by improving success rates and decreasing complications.

Excitement surrounding this technology continues to grow among hospitalists, leading to a proliferation of high-quality educational programs over the last 5 years. Most notable among these offerings has been the more comprehensive training available through the Society of Hospital Medicine (SHM) certificate-based pathway, though many other strong options exist, including institution-based curricula, such as the HealthPartners CHAMP program, ${ }^{3}$ and pediatric-focused programs. Growth in training is also occurring among medical students and residents. As of a 2012 survey, the majority (51\%) of US medical schools had begun to weave ultrasound into their curricula ${ }^{4}$ and this growth is also occurring in internal medicine and pediatric residency programs. ${ }^{5}$

Given the high potential for this technology and the growth in interest, it is an excellent time to pause and review some of the challenges faced by practitioners, hospitalist groups, and educators seeking to optimize POCUS implementation. A deliberate approach to POCUS education, the development of shared standards for high-quality use, and an ongoing dedication to develop specialty-specific practices will largely determine how much of this potential is fulfilled.

The largest challenge is likely to be educational. Educating clinicians to be able to integrate POCUS into practice is a complex, multistep process requiring not only an adequate core of didactic training and access to machines, but also the structured opportunity to develop rudimentary hands-on skills. Such initial training should be followed by continued practice and feedback as developing POCUS users progress toward independent practice. The study by Kumar et al. ${ }^{6}$ reaffirms that brief didactic lectures and access to machines are necessary,

*Corresponding Author: Daniel Schnobrich, MD; Email: Schn0364@umn.edu; Telephone: 612.624 .0579

Received: December 9, 2019; Revised: January 10, 2019;

Accepted: January 13, 2020

๑ 2020 Society of Hospital Medicine DOI 10.12788/jhm.3384 but they are clearly insufficient for learners to be able to use POCUS independently for a wide variety of applications. Their intervention also contrasts markedly with the 20 hours of didactics and 150 supervised scans recommended by the American College of Emergency Physicians prior to independent use for a core of six applications.?

Shared standards for education, use, and oversight will be crucial to fulfilling the potential of POCUS within hospital medicine. Our belief is that much can be learned from the thoughtful approach taken during the development of POCUS as a mainstream tool in emergency medicine in the early 2000s. In this approach, emergency physicians determined a sufficient and achievable standard of training for core POCUS applications, which was widely adopted. Based on completion of this training, physicians who were required to complete credentialing from their hospitals were widely able to achieve it, without any need for external certification. Emergency medicine guidelines further mandated the documentation of examinations and the creation of an exam report, features that improve clinical communication and facilitate quality improvement. Quality assurance processes that reviewed images and clinician interpretations were established as mandatory, which they should be in hospital medicine. Evidence was produced as to which exams physicians could do reliably with this focused training and which they could not. In the context of these thoughtful constructs, lawsuits have been noted to be exceedingly rare; and when they do occur, they have typically been for the failure to use POCUS rather than the converse. ${ }^{8}$

While many of these precepts deserve replication, others should also be modified to reflect changes in technology, medical education, and medical practice over the last 20 years and to improve upon this base of success. For example, with POCUS training now appearing in many medical school and residency curricula, training paradigms for both residents and attendings will need to accommodate a wider range of incoming skills. Emphasis should continue to be shifted toward competency-based assessments and entrustment and away from a fixed training time or exam number threshold. Important financial aspects have also changed. The cost of practical machines has dropped considerably, and medicine is shifting away from a fee-for-service model. While it remains appropriate that physicians may bill for POCUS examinations, it is likely that improved diagnosis, improved throughput, and a reduction in complications will yield greater value and should be the emphasis of cost/value discussions. ${ }^{9}$ Finally, while hospitals may impose credentialing, this process can also create a burden not present for most other noninvasive skills and may deter appropriate use. If this approach is chosen by a hospital, 
requirements should ideally remain modest, and as these skills become more widespread, POCUS should ultimately be built into board examinations and core credentialing. ${ }^{9}$

Thoughtful and concerted effort will be required by hospitalist leaders, educational innovators, and professional societies in developing POCUS to best serve hospitalists and their patients. This work has already begun. For example, in 2019 SHM offered a position statement outlining important aspects such as current evidence-based applications, training pathways, quality assurance, and program management. ${ }^{10}$ These recommendations should guide both adult and pediatric hospitalists. The Alliance for Academic Internal Medicine offered a similar position statement for resident training. ${ }^{11}$ Interest groups are growing in numerous professional societies, which will facilitate collaboration and promote propagation of best practices. High-quality educational tools are continuing to be developed by numerous organizations.

While further development is needed to add the detail, granularity, and practical tools that educational and practice leaders need to assure that POCUS achieves its potential in hospital medicine, the foundation for POCUS use within the specialty is being thoughtfully constructed. As this process proceeds, it will be vital to continue to learn from our emergency medicine colleagues, who have already met similar challenges, while at the same time be able to develop a modern POCUS model optimized for hospital medicine workflow, training, and patient care.

Disclosures: The authors have nothing to disclose.

\section{References}

1. Kinnear B, Kelleher M, Chorny V. Clinical practice update: Point-of-care ultrasound for the pediatric hospitalist. J Hosp Med. 2019;15(3):170-172. https:// doi.org/10.12788/jhm.3325.

2. Kelleher M, Kinnear B, Olson A. Clinical progress note: Point-of-care ultrasound in the evaluation of the dyspneic adult. J Hosp Med. 2020;15(3):173175. https://doi.org/10.12788/jhm.3340.

3. Mathews BK, Reierson K, Vuong K, et al. The design and evaluation of the Comprehensive Hospitalist Assessment and Mentorship with Portfolios (CHAMP) Ultrasound Program. J Hosp Med. 2018;13(8):544-550. https://doi. org/10.12788/jhm.2938.

4. Bahner DP, Goldman E, Way D, Royall NA, Liu YT. The state of ultrasound education in U.S. medical schools: Results of a national survey. Acad Med. 2014;89(12):1681-1686. https://doi.org/10.1097/ACM.0000000000000414.

5. Reaume M, Siuba M, Wagner M, Woodwyk A, Melgar TA. Prevalence and Scope of point-of-care ultrasound education in internal medicine, pediatric, and medicine-pediatric residency programs in the United States. J Ultrasound Med. 2019;38(6):1433-1439. https://doi.org/10.1002/jum.14821.

6. Kumar A, Weng Y, Wang $L$, et al. Portable ultrasound device usage and learning outcomes among internal medicine trainees: a parallel-group randomized trial. J Hosp Med. 2020;15(3):154-159. https://doi.org/10.12788/ jhm.3351.

7. Ultrasound Guidelines: Emergency, Point-of-Care and Clinical Ultrasound Guidelines in Medicine. Ann Emerg Med. 2017;69(5):e27-e54. https://doi. org/10.1016/j.annemergmed.2016.08.457.

8. Stolz L, O'Brien KM, Miller ML, Winters-Brown ND, Blaivas M, Adhikari S. A review of lawsuits related to point-of-care emergency ultrasound applications. West J Emerg Med. 2015;16(1):1-4. https://doi.org/10.5811/westjem.2014.11.23592.

9, Soni NJ, Tierney DM, Jensen TP, Lucas BP. Certification of Point-of-Care Ultrasound Competency. J Hosp Med. 2017;12(9):775-776. doi:10.12788/ jhm.2812

10. Soni NJ, Schnobrich D, Matthews BK, et al. Point-of-Care Ultrasound for hospitalists: A position statement of the society of hospital medicine. J Hosp Med. 2019;14. https://doi.org/10.12788/jhm.3079.

11. LoPresti CM, Jensen TP, Dversdal RK, Astiz DJ. Point-of-Care Ultrasound for Internal Medicine Residency Training: A position statement from the Alliance of Academic Internal Medicine. Am J Med. 2019 Nov;132(11):1356-1360. https://doi.org/10.1016/j.amjmed.2019.07.019 